\title{
A Case on Streptococcal Pneumonia Associated with Leptomeningitis, Osteomyelitis and Epidural Abscess in a Patient with AIDS
}



\author{
Jae Woong Jeon, M.D., Hee Jung Yoon, M.D., Ph.D., Joo Seok Kim, M.D., Il Hwan Ryu, M.D., Ji \\ Wook Choi, M.D., Min Gyu Kim, M.D., Young Min Na, M.D. and Hyeon Jeong Yun, M.D. \\ Division of Infectious Diseases, Department of Internal Medicine, Eulji University Hospital, Eulji University School of Medicine, \\ Daejeon, Korea
}

Patients with acquired immunodeficiency syndrome (AIDS) are at higher risks of bacterial pneumonia than the general population, and the pathogen is the most commonly involved Streptococcus pneumoniae. We hereby report a case of pneumococcal pneumonia associated with leptomeningitis, osteomyelitis and epidural abscess in a patient with AIDS. He is being successfully treated with ampicillin/sulbactam and clindamycin. And because the pneumococcal infection is usually associated with morbidity and mortality rates in the setting of AIDS, we should consider for pneumococcal vaccinations among the AIDS populations.

Keywords: Acquired Immunodeficiency Syndrome; Streptococcus; Pneumonia; Meningitis; Osteomyelitis

\section{Introduction}

Patients with acquired immunodeficiency syndrome (AIDS) are exposed to multiple opportunistic infections. In the United States, the most common AIDS-related opportunistic infections recorded were Pneumocystis carinii pneumonia, Mycobacterium avium complex disease and esophageal can-

Address for correspondence: Hee Jung Yoon, M.D., Ph.D.

Division of Infectious Diseases, Department of Internal Medicine, Eulji University Hospital, Eulji University School of Medicine, 95 Dunsanseoro, Seo-gu, Daejeon 302-799, Korea

Phone: 82-42-611-3096, Fax: 82-42-611-3853

E-mail:yhj822@medimail.co.kr

Received: Aug. 20, 2013

Revised: Sep. 6, 2013

Accepted: Sep. 26, 2013

(c) It is identical to the Creative Commons Attribution Non-Commercial License (http://creativecommons.org/licenses/by-nc/3.0/).

Copyright (c) 2014

The Korean Academy of Tuberculosis and Respiratory Diseases.

All rights reserved. didiasis ${ }^{1}$, while in Thailand, tuberculosis was the most common cause. In Korea, the incidence of candidiasis has been reported as being higher than in the West ${ }^{2}$, and in recent years, AIDS patients with esophageal stenosis caused by candidiasis and retroperitoneal tuberculosis lymphadenopathy have been reported. In sub-Saharan Africa, tuberculosis, bacterial infections and malaria were the most common serious infections diagnosed in individuals with AIDS, and among bacterial infections a high prevalence of Streptococcus pneumoniae infection was found ${ }^{3}$.

One of risk factors for pneumococcal infection is an immune deficiency disease (e.g., human immunodeficiency virus $[\mathrm{HIV}])$. And pneumococcal infection is associated with morbidity and mortality in patients with AIDS. We report the case of 53-year-old Korean male with AIDS and pneumococcal pneumonia in addition to leptomeningitis, osteomyelitis, and epidural abscess. He was successfully treated with appropriate antibiotics.

\section{Case Report}

A 53-year-old male presented with cough, sputum, and 
headache for 2 weeks.

Five years previously, he had been diagnosed with HIV. He noted the onset of cough, sputum, and headache. The cough worsened, his sputum turned more yellowish and headaches accompanied by neck stiffness were related to coughing.

Over the first five years following the diagnosis of HIV, he was treated with antiretroviral medications (lamivudine and zidovudine; Combivir, Ritonavir; Norvir, atazanavir sulfate; Reyataz).

\section{Physical examination}

Vital signs were as follows: temperature, $38.0^{\circ} \mathrm{C}$; blood pressure, 110/70 mm Hg; pulse rate, 90/min; respiratory rate, 20/ min. He was alert mentally. Auscultation revealed rales in the right lower lung field. A Brudzinski-Kernig test was positive.

\section{Laboratory analysis}

Complete blood count results were white blood cell
$18.43 \times 10^{3} / \mu \mathrm{L}$ (neutrophils $91.3 \%$, lymphocytes $3.5 \%$ ), hemoglobin $14.0 \mathrm{~g} / \mathrm{dL}$, hematocrit $40.3 \%$, and platelets $290 \times 10^{3} /$ $\mu \mathrm{L}$. Serum chemistry showed blood urea nitrogen $14 \mathrm{mg} /$ $\mathrm{dL}$, creatinine $0.66 \mathrm{mg} / \mathrm{dL}$, aspartate aminotransferase $25 \mathrm{IU} /$ $\mathrm{L}$, alanine aminotransferase $23 \mathrm{IU} / \mathrm{L}$, total bilirubin $1.92 \mathrm{mg} /$ $\mathrm{dL}$, and C-reactive protein $38.51 \mathrm{mg} / \mathrm{dL}$. Cerebral spinal fluid (CSF) showed a white blood cell count of $108 / \mu \mathrm{L}$, red blood cell count $0 / \mu \mathrm{L}$, glucose $53 \mathrm{mg} / \mathrm{dL}$, and protein $123 \mathrm{mg} / \mathrm{dL}$. CSF and sputum bacterial cultures were negative. A CD4 Tcell count of $139 / \mu \mathrm{L}$ and HIV RNA of 20.7 copies $/ \mathrm{mL}$ were recorded.

\section{Radiological findings}

Plain chest radiography showed ground glass opacity and consolidation in both lung fields (especially the right lower) (Figure 1A). Contrast-enhanced chest computed tomography showed multiple irregular nodular infiltration in both lungs and patchy consolidation with ground glass opacity of the right middle and lower lung (Figure 1B). Contrast-enhanced
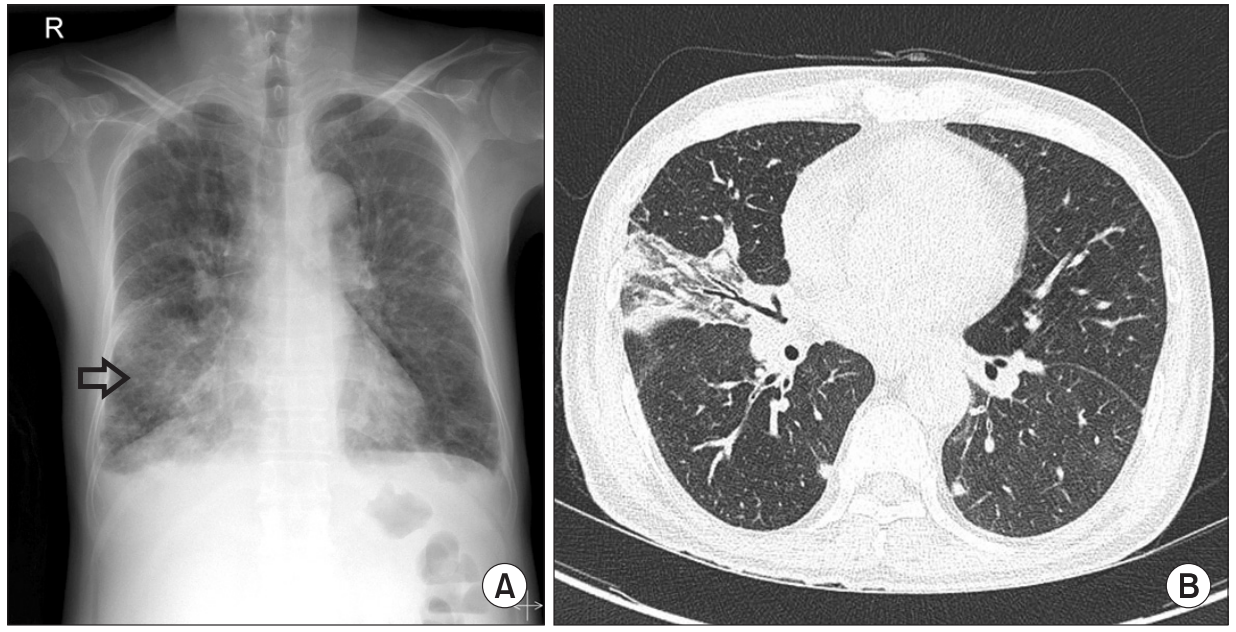

Figure 1. Initial lung images. (A) Initial chest X-ray showed ground glass opacity and consolidation on both lung fields (especially the right lower lung field). (B) Initial contrast-enhanced chest computed tomography showed multiple irregular nodular infiltration in both lungs and patchy consolidation with ground glass opacity in the right middle and lower lungs.
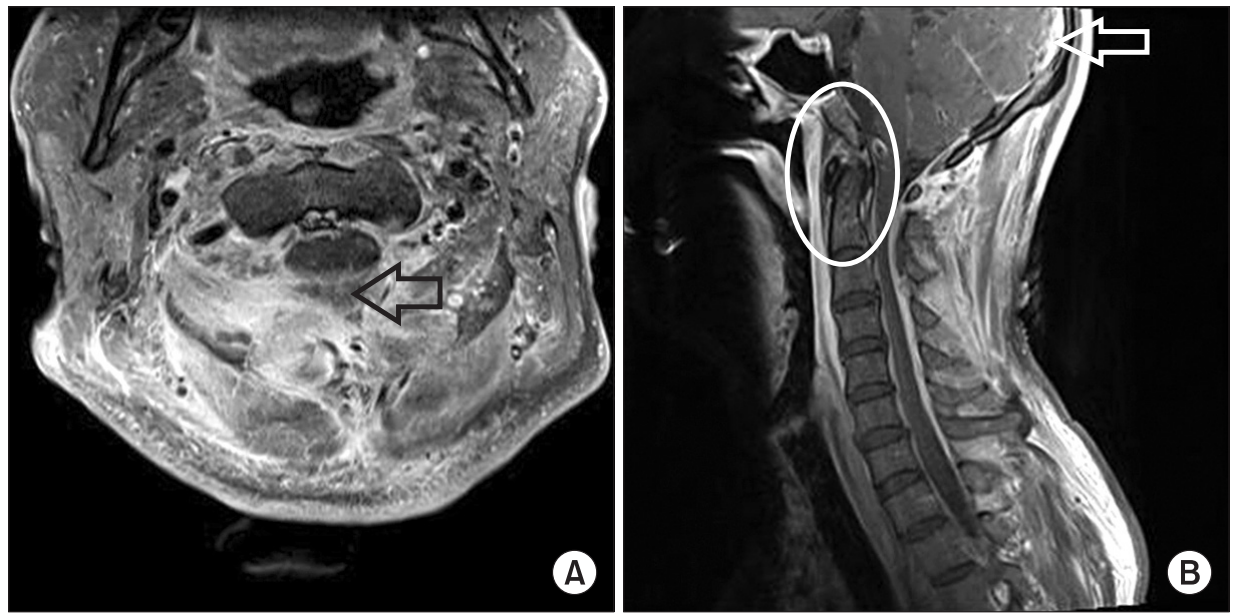

Figure 2. Contrast-enhanced cervical spine magnetic resonance imaging. (A) Posterior epidural abscess; peripheral rim enhancing lesion at the posterior epidural space of cervical spine (C2-3) (arrow). (B) Osteomyelitis; mild enhancement in the clivus and scanned cervical spine (circle), early leptomeningitis; prominent leptomeningeal enhancement (arrow). 

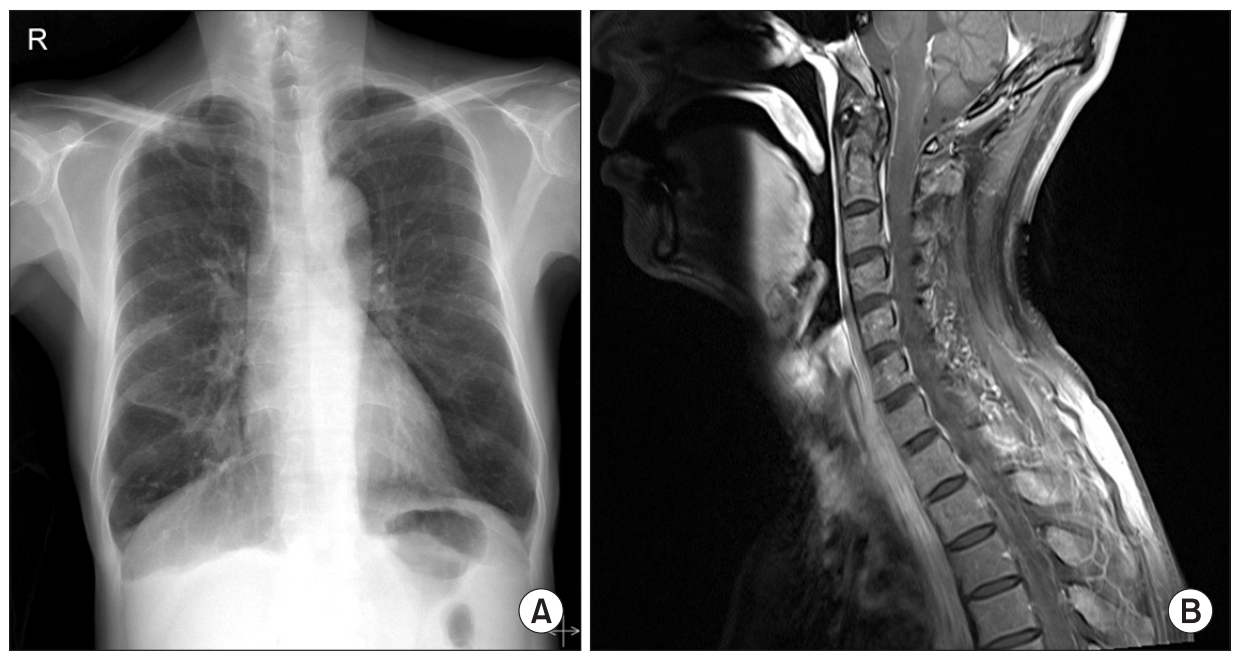

Figure 3. Follow-up of chest X-ray and cervical spine magnetic resonance. (A) Decreased ground glass opacity and consolidation on both lung fields were noted. (B) There is no abnormal signal nor contrast enhancement in C-spine and leptomeninges.

cervical spine magnetic resonance imaging (MRI) showed a posterior epidural abscess: a peripheral rim-enhancing lesion at the posterior C2-3 epidural space (Figure 2A); osteomyelitis; mild scanning enhancement of the clivus and cervical spine; early leptomeningitis; and prominent leptomeningeal enhancement (Figure 2B).

\section{Treatment and prognosis}

The patient was administered vancomycin ( $1 \mathrm{~g} \mathrm{q} 12 \mathrm{hr}$ IV), ceftriaxone ( 2 g q $24 \mathrm{hr}$ ), clindamycin (600 mg q $12 \mathrm{hr}$ ) and fluconazole ( $400 \mathrm{mg} \mathrm{q} 24 \mathrm{hr}$ ). The patient's fever reduced 3 days following admission. His epidural abscess was discussed with the neurosurgeon in our hospital. There were no neurological symptoms, no surgical treatment was performed and antibiotic therapy was maintained. At follow-up 5 days after admission, a plain chest radiograph showed improvement, and S. pneumoniae was detected in two blood cultures that showed sensitivity to most antibiotics. The patient was thus switched from vancomycin plus ceftriaxone to ampicillin (2 g) plus sulbactam ( 1 g q 6 hr IV). Fifteen days after admission, neck stiffness had improved and cough and sputum production reduced. A plain chest X-ray showed decrease in both ground glass opacity and consolidation in both lung fields (Figure 3A). Twenty-six days after admission, laboratory analysis was as follows: white blood cell count $5.42 \times 10^{3} / \mu \mathrm{L}$, neutrophils $55.9 \%$, lymphocytes $25.9 \%$, and C-reactive protein $0.26 \mathrm{mg} / \mathrm{dL}$. The patient wished to be discharged, so antibiotic therapy was switched from intravenous to oral (amoxicillin/ clavulanate $625 \mathrm{mg}$ PO tid). Since discharge, no recurrence of symptoms or evidence of pneumonia according to plain chest radiography have been observed. One year after discharge, there is no abnormal finding on cervical MRI (Figure 3B).

\section{Discussion}

In patients with AIDS, the lung is the site most frequently affected by the disease and, currently, the most frequent pulmonary manifestation in developed countries is bacterial (especially pneumococcal) pneumonia, with the next most frequent being Pneumocystis jiroveci pneumonia. Clinical symptoms include fever, cough and the production of purulent sputum. The symptoms are similar in bacterial and Pneumocystis pneumonia, but acute onset of symptoms suggests a bacterial process ${ }^{4}$. Factors that increase the risk of invasive pneumococcal disease include alcohol abuse, drug addiction and an HIV-infected population. The literature includes one case of spinal epidural abscess with myelitis and meningitis caused by S. pneumoniae in a young child ${ }^{5}$. In Korea, one fatal case of $S$. pneumoniae infection associated with AIDS was reported $^{6}$.

The incidence of bacterial meningitis has dropped dramatically in the general population since the introduction of vaccines effective against bacterial pathogens such as $S$. pneumoniae, Neisseria gonorrhoeae, and Haemophilus influenzae. However, S. pneumoniae is the most common strain involved in bacterial meningitis in patients with AIDS, with an incidence 150 -fold that of the general population. The therapeutic regimen of choice for pneumococcal meningitis in adults with AIDS is ampicillin plus ceftazidime plus vancomycin. Adjuvant therapy with corticosteroids reduces mortality among adults in the general population, but is not recommend for patients with AIDS?

The mortality rate for osteomyelitis is over $20 \%$ in patients with AIDS. In particular, this rate is higher in patients with both AIDS and one or more artificial joints. In most cases of osteomyelitis in patients with AIDS, pathogens were disseminated from septic arthritis, the most common being Staphylococcus aureus and S. pneumoniae. MRI is the most sensitive and specific method for the detection of osteomyelitis (sensitivity 
$82 \%$ to $100 \%$, specificity $75 \%$ to $96 \%)^{8}$. Definitive diagnosis is made by bone biopsy and culture, and blood cultures may be positive in cases of bacterial osteomyelitis resulting from haematogenous spread. In addition to surgical treatment, cases of acute osteomyelitis can be treated with antibiotic therapy alone, and the key to successful management is early diagnosis $^{9,10}$.

Spinal epidural abscess empyema is a rare condition that usually occurs secondary to metastatic infection such as osteomyelitis, and is more common in patients with AIDS. Therefore, in patients with spinal infection, immunosuppressive diseases such as AIDS should also be considered. Diagnosis of spinal infection requires the analysis of cerebrospinal fluid and MRI. An early surgical approach should be considered in the event of complication by compressive myelopathy ${ }^{8}$.

In the present case, who had no history of pneumococcal vaccination, the patient presented with upper respiratory symptoms diagnosed as pneumonia complicated by leptomeningitis, osteomyelitis and epidural abscess, as confirmed by MRI. S. pneumoniae, as detected by blood culture, can cause upper and lower respiratory tract infections, meningitis, bacteraemia and/or invasive infections. A review of the literature allows identification of the main risk factors: congestive cardiac failure, diabetes mellitus, chronic lung disease and immunodeficiency (including those with AIDS). Infection due to S. pneumoniae is more frequent and more severe in such patients ${ }^{11}$. In regard to treatment of streptococcal infections, patients with AIDS receive the same as the general population, but AIDS is the main risk factor for streptococcal infections and mortality is high in these patients compared with the general population. Therefore, HIV-infected adults with a CD4 ${ }^{+}$ count of $>200$ cells $/ \mu \mathrm{L}$ should be administered a single dose of 23-valent polysaccharide pneumococcal vaccine unless they had received this vaccine during the previous 5 years ${ }^{12}$. One recent report discusses the protective effect of 7 -valent conjugated vaccine in severe pneumococcal infections in HIVinfected patients ${ }^{13}$. Community-acquired pneumonia caused by $S$. pneumoniae is an important public health problem, especially in immunosuppressive diseases such as AIDS. Highly active antiretroviral therapy has led to improved survival rates in patients with AIDS, and thus vaccination is recommended to reduce the incidence of pneumococcal infections in patients with AIDS. More research is needed on the effects of vaccination.

\section{References}

1. Kaplan JE, Hanson D, Dworkin MS, Frederick T, Bertolli J, Lindegren ML, et al. Epidemiology of human immunodeficiency virus-associated opportunistic infections in the United States in the era of highly active antiretroviral therapy. Clin Infect Dis 2000;30 Suppl 1:S5-14.

2. Kim JM, Cho GJ, Hong SK, Chung JS, Jang KH, Kim CO, et al. Epidemiologic and clinical features of HIV infection/AIDS in Koreans. Korean J Med 2001;61:355-64.

3. Holmes CB, Losina E, Walensky RP, Yazdanpanah Y, Freedberg KA. Review of human immunodeficiency virus type 1-related opportunistic infections in sub-Saharan Africa. Clin Infect Dis 2003;36:652-62.

4. Goldman L, Schafer AI. Cecil medicine. 24th ed. Philadelphia: Saunders Elsevier; 2011.

5. Bhattacharya M, Joshi N. Spinal epidural abscess with myelitis and meningitis caused by Streptococcus pneumoniae in a young child. J Spinal Cord Med 2011;34:340-3.

6. Lee MS, Woo JH, Kim BN, Kim EO, Kim YS, Ryu JS. A case of Streptococcus pneumoniae infection with AIDS: an autopsy report. Korean J Infect Dis 1996;28:269-75.

7. Scarborough M, Gordon SB, Whitty CJ, French N, Njalale Y, Chitani A, et al. Corticosteroids for bacterial meningitis in adults in sub-Saharan Africa. N Engl J Med 2007;357:2441-50.

8. Richie MB, Pruitt AA. Spinal cord infections. Neurol Clin 2013;31:19-53.

9. Pineda C, Vargas A, Rodriguez AV. Imaging of osteomyelitis: current concepts. Infect Dis Clin North Am 2006;20:789-825.

10. Lew DP, Waldvogel FA. Osteomyelitis. Lancet 2004;364:36979 .

11. Chidiac C. Pneumococcal infections and adult with risk factors. Med Mal Infect 2012;42:517-24.

12. Kaplan JE, Benson C, Holmes KK, Brooks JT, Pau A, Masur $\mathrm{H}$, et al. Guidelines for prevention and treatment of opportunistic infections in HIV-infected adults and adolescents: recommendations from CDC, the National Institutes of Health, and the HIV Medicine Association of the Infectious Diseases Society of America. MMWR Recomm Rep 2009;58:1-207.

13. French N, Gordon SB, Mwalukomo T, White SA, Mwafulirwa G, Longwe $\mathrm{H}$, et al. A trial of a 7-valent pneumococcal conjugate vaccine in HIV-infected adults. N Engl J Med 2010;362:812-22. 